\title{
Coupled waves: a powerful concept in modern optics
}

Rene Daendliker

Rene Daendliker, "Coupled waves: a powerful concept in modern optics," Proc. SPIE 3190, Fifth International Topical Meeting on Education and Training in Optics, (8 December 1997); doi: 10.1117/12.294394

Event: Fifth International Topical Meeting on Education and Training in Optics, 1997, Delft, Netherlands 


\title{
Coupled Waves: A Powerful Concept in Modern Optics
}

\author{
René Dändliker \\ Institute of Microtechnology, University of Neuchâtel, Breguet 2, CH-2000 Neuchâtel, Switzerland \\ E-mail: dandliker@imt.unine.ch
}

\begin{abstract}
Coupled waves are used in many fields of modern optics: volume holograms, Bragg reflectors, acousto-optic modulators, waveguide couplers, distributed feedback lasers, polarization effects in optical fibers and liquid crystals, non-linear optics, parametric amplifiers and oscillators, 4-wave mixing, etc. First, the general concept of coupled waves is introduced for any kind of perturbation (spatial and temporal) of the dielectric polarization within an optical medium. The result is a general form of coupled wave solutions. Second, the particular topics mentioned above are treated by introducing the special conditions for the basic modes to be considered (plane waves, guided waves, optical polarizations) and the physical effects relating the induced perturbation of the dielectric polarization to the electrical field (spatial and temporal variation of refractive index, absorption or birefringence, non-linear optical effects, etc.). This approach puts the emphasis on teaching concepts rather than presenting particular effects. The fundamental role of phase-matching (Bragg) and the similarities of the solutions for different physical effects emerge clearly.
\end{abstract}

Keywords: coupled waves, phase-matching, volume holograms, acousto-optic modulators, waveguide couplers, polarization effects in optical fibers, non-linear optics

\section{INTRODUCTION}

Coupled waves are used in many fields of modern optics: volume holograms, Bragg reflectors, acousto-optic modulators, waveguide couplers, distributed feedback lasers, polarization effects in optical fibers and liquid crystals, non-linear optics, parametric amplifiers and oscillators, 4-wave mixing, etc. In optics textbooks and courses, the coupled wave approach is usually introduced ad hoc when needed in one of the particular chapters mentioned above. ${ }^{1,2} \mathrm{~A}$ few years ago I started a course on coupled waves in modern optics.

First, the general concept of coupled waves is introduced for any kind of perturbation (spatial and temporal) of the dielectric polarization within an optical medium. Assuming that a set of orthogonal solutions (modes) is known for the situation without perturbation, a general form of coupled wave solutions can be developed. In the case of weak coupling (negligible second derivatives) these solutions take the form of projection integrals (generalized scalar products) of the dielectric perturbation and the electrical field of the considered modes.

Second, the particular topics mentioned above are treated by introducing the special conditions for the basic modes to be considered (plane waves, guided waves, optical polarizations) and the physical effects relating the induced perturbation of the dielectric polarization to the electrical field (spatial and temporal variation of refractive index, absorption, birefringence, nonlinear optical effects, etc.). The well know solutions for different boundary conditions are then developed and discussed.

This approach puts the emphasis on teaching concepts rather than presenting particular effects. The fundamental role of phase-matching (Bragg) and the similarities of the solutions for different physical effects emerge clearly. Coupled mode equations are also well suited for numerical evaluation. These are the reasons why I believe that introducing coupled waves as a concept in modern optics is most adequate for education in optics at a university level.

\section{THE CONCEPT OF COUPLED WAVES}

The concept of coupled waves is used to describe the propagation of optical waves in a dielectric medium which can be characterized by any kind of perturbation (spatial and temporal) of the dielectric polarization with respect to a basic situation with known solutions. The concept of coupled waves is a method to find (approximate) solutions of the wave equation (for the electric field vector) assuming a perturbation induced by the dielectric polarization of the medium. In the following, a general form of coupled wave solutions will be developed for the case of weak coupling. 


\subsection{Wave propagation in a dielectric medium}

Light as an electro-magnetic wave is described by the propagation of the electric and the magnetic field vectors $\mathbf{E}(\mathbf{x}, \mathbf{t})$ and $\mathbf{H}(\mathbf{x}, \mathbf{t})$ in space $\mathbf{x}$ and time t. The starting point are Maxwell's equations

$$
\operatorname{rot} \mathbf{E}+\dot{\mathbf{B}}=\mathbf{0}, \quad \operatorname{div} \mathbf{B}=0, \quad \operatorname{div} \mathbf{D}=\rho, \quad \operatorname{rot} \mathbf{H}=\dot{\mathbf{D}}+\mathbf{i},
$$

where $\rho(x, t)$ and $\mathbf{i}(\mathbf{x}, \mathbf{t})$ are the electric charge and the current density. The electric and magnetic displacement field vectors $\mathbf{D}(\mathbf{x}, \mathbf{t})$ and $\mathbf{B}(\mathbf{x}, \mathbf{t})$ are in general given by

$$
\mathbf{D}(\mathbf{x}, \mathrm{t})=\varepsilon_{0} \mathbf{E}(\mathbf{x}, \mathrm{t})+\mathbf{P}(\mathbf{x}, \mathrm{t}), \quad \mathbf{B}(\mathbf{x}, \mathbf{t})=\mu_{0}[\mathbf{H}(\mathbf{x}, \mathbf{t})+\mathbf{M}(\mathbf{x}, \mathrm{t})],
$$

where $\mathbf{P}(\mathbf{x}, \mathbf{t})$ and $\mathbf{M}(\mathbf{x}, \mathrm{t})$ are the dielectric and the magnetic polarizations of the medium, respectively, and $\varepsilon_{0}$ and $\mu_{0}$ are the electric and the magnetic permeabilities of the vacuum.

A transparent dielectric medium is characterized by $\rho=0, \mathbf{i}=\mathbf{0}, \mathbf{M}=\mathbf{0}$, and $\mathbf{D}=\varepsilon_{0} \mathbf{E}+\mathbf{P}$. From Maxwell's equations (1) we get then the wave equation for the electric field vector in the general form

$$
\Delta \mathbf{E}(\mathbf{x}, \mathrm{t})=\mu_{0} \varepsilon_{0} \ddot{\mathbf{E}}(\mathbf{x}, \mathbf{t})+\mu_{0} \ddot{\mathbf{P}}(\mathbf{x}, \mathrm{t}) .
$$

The dielectric polarization $\mathbf{P}(\mathbf{x}, t)$ is now considered to be composed of two parts: $\mathbf{P}_{0}(\mathbf{x}, \mathrm{t})$ for which at least some solutions of the wave equation are known and a perturbation $\mathbf{p}(\mathbf{x}, \mathbf{t})$. This perturbation is supposed to be weak, i.e. $|\mathbf{p}(\mathbf{x}, \mathbf{t})|<<\varepsilon_{0}|\mathbf{E}(\mathbf{x}, \mathbf{t})|$. In an originally isotropic and linear material, $\mathbf{P}(\mathbf{x}, \mathbf{t})$ can be written as

$$
\mathbf{P}(\mathbf{x}, \mathrm{t})=\mathbf{P}_{0}(\mathbf{x}, \mathrm{t})+\mathbf{p}(\mathbf{x}, \mathrm{t})=\varepsilon_{0} \chi(\mathbf{x}) \mathbf{E}(\mathbf{x}, \mathrm{t})+\mathbf{p}(\mathbf{x}, \mathrm{t})
$$

where $\chi(\mathbf{x})$ is the electric susceptibility. Inserting $\mathbf{P}(\mathbf{x}, \mathbf{t})$ into Eq. (3) and using the relations $c^{2}=1 / \mu_{0} \varepsilon_{0}$ and $\varepsilon(\mathbf{x})=1+\chi(\mathbf{x})$ yields

$$
\Delta \mathbf{E}(\mathbf{x}, \mathbf{t})-(1 / \mathrm{c})^{2} \varepsilon(\mathbf{x}) \ddot{\mathbf{E}}(\mathbf{x}, \mathbf{t})=\mu_{0} \ddot{\mathbf{p}}(\mathbf{x}, \mathrm{t}) .
$$

Equation (5) describes the wave propagation in a medium with perturbation $\mathbf{p}(\mathbf{x}, \mathbf{t})$. Without perturbation, Eq. (5) becomes

$$
\Delta \mathbf{E}(\mathbf{x}, \mathrm{t})-(1 / \mathrm{c})^{2} \varepsilon(\mathbf{x}) \ddot{\mathbf{E}}(\mathbf{x}, \mathrm{t})=\mathbf{0},
$$

where $\varepsilon(\mathbf{x})$ may represent the refractive index of a homogeneous material or the profile of a wave guide or an optical fiber.

\subsection{Solution of wave propagation by coupled modes}

It is now assumed, that at least some solutions of the wave equation without perturbation (6) are known and that they can be described as modes propagating in the z-direction of a Cartesian coordinate system (Fig. 1). These modal solutions of Eq. (6) are of the form

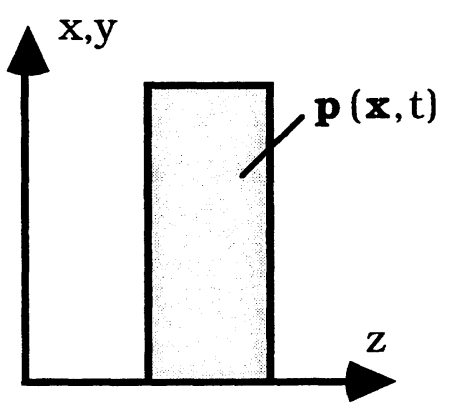

Fig. 1. Geometry for coupled mode solution.

$$
\mathbf{E}_{n}(\mathbf{x}, \mathrm{t})=\mathbf{U}_{\mathrm{n}}(\mathbf{x}, \mathbf{y}) \mathrm{e}^{\mathrm{i}\left(\omega_{\mathrm{n}} \mathrm{t}-\boldsymbol{\beta}_{\mathrm{n}} \mathrm{z}\right)} .
$$

The electric field distribution $\mathbf{U}_{\mathrm{n}}(\mathrm{x}, \mathrm{y})$ of the modal solutions are independent of $\mathrm{z}$ and the relations between the propagation constants $\beta_{n}$ and the angular frequencies $\omega_{\mathrm{n}}$ are given by Eq. (6). Typical solutions are plane waves and modes of optical wave guides and fibers.

The coupled wave approach assumes now, that the electric field $\mathbf{E}(\mathbf{x}, \mathbf{t})$ in the perturbed medium can be obtained by a linear superposition of an appropriate selection of $\mathrm{N}$ basic modes, namely

$$
\mathbf{E}(x, t)=\sum_{n=1}^{N} A_{n}(z) E_{n}(x, t)=\sum_{n=1}^{N} A_{n}(z) U_{n}(x, y) e^{i\left(\omega_{n} t-\beta_{n} z\right)},
$$

where $A_{n}(z)$ are complex coefficients describing the amplitudes and phases of the different coupled modes $\mathbf{E}_{\mathbf{n}}$. 
Introducing now Eq. (8) into Eq. (5) yields

$$
\sum_{n=1}^{N}\left[A_{n} \Delta \mathbf{E}_{n}+2 \frac{d A_{n}}{d z} \frac{\partial \mathbf{E}_{n}}{\partial z}+\frac{d^{2} A_{n}}{d z^{2}} \mathbf{E}_{n}-(1 / c)^{2} \varepsilon(\mathbf{x}) A_{n} \ddot{\mathbf{E}}_{n}\right]=\mu_{0} \ddot{\mathbf{p}}(\mathbf{x}, t)
$$

Because the modes $\mathbf{E}_{\mathrm{n}}$ satisfy the wave equation without perturbation (6), Eq. (9) reduces to

$$
\sum_{n=1}^{N}\left[2 \frac{d A_{n}}{d} \frac{\partial \mathbf{E}_{n}}{\partial z}+\frac{d^{2} A_{n}}{d z^{2}} \mathbf{E}_{n}\right]=\sum_{n=1}^{N}\left[-2 i \beta_{n} \frac{d A_{n}}{d z} \mathbf{E}_{n}+\frac{d^{2} A_{n}}{d z^{2}} \mathbf{E}_{n}\right]=\mu_{0} \ddot{\mathbf{p}}(\mathbf{x}, \mathrm{t})
$$

\subsection{Orthogonality of the selected modes}

Assuming orthogonality of the $N$ selected modes $\mathbf{E}_{n}(\mathbf{x}, \mathrm{t})$, Eq. (10) can be separated into a set of $\mathrm{N}$ differential equations for the amplitudes $A_{n}(z)$. The general expression for the orthogonality reads

$$
\frac{1}{\mathrm{~T}} \int_{0}^{\mathrm{T}} \mathrm{dt} \int_{-\infty}^{+\infty} \mathrm{dx} d y \mathbf{E}_{\mathrm{n}}(\mathbf{x}, \mathrm{t}) \mathbf{E}_{\mathrm{m}}^{*}(\mathbf{x}, \mathrm{t})=\mathrm{e}^{-\mathrm{i}\left(\beta_{\mathrm{n}}-\beta_{\mathrm{m}}\right) \mathrm{z}} \frac{1}{\mathrm{~T}} \int_{0}^{\mathrm{T}} \mathrm{dt} \mathrm{e}^{\mathrm{i}\left(\omega_{\mathrm{n}}-\omega_{\mathrm{m}}\right) \mathrm{t}} \int_{-\infty}^{+\infty} \mathrm{dx} d y \mathbf{U}_{\mathrm{n}}(\mathrm{x}, \mathrm{y}) \mathbf{U}_{\mathrm{m}}^{*}(\mathrm{x}, \mathrm{y})=\mathrm{W}_{\mathrm{m}} \delta_{\mathrm{mn}},
$$

where $T$ is the integration time and $W_{m}$ is the value of the normalized energy of each mode. There are two possibilities to fulfill Eq. (11): either the frequencies of the modes are different $\left(\omega_{n} \neq \omega_{m}\right)$ or the modes are spatially orthogonal (i.e., $U_{n}(x, y)$ and $U_{m}(x, y)$ are orthogonally polarized or the shape of the modes is orthogonal). Using the orthogonality relation (11) we get from Eq. (10) a set of $\mathrm{N}$ differential equations

$$
2 i \beta_{m} \frac{d A_{m}}{d z}+\frac{d^{2} A_{m}}{d z^{2}}=\frac{\mu_{0}}{T W_{m}} e^{i \beta_{m} z} \int_{-\infty}^{+\infty} d x d y \int_{0}^{T} d t U_{m}^{*}(x, y) e^{-i \omega_{m} t} \ddot{p}(x, t) .
$$

In the case of weak coupling, defined by

$$
\frac{\mathrm{dA}_{\mathrm{m}}}{\mathrm{d}}>>\frac{1}{\beta_{\mathrm{m}}} \frac{\mathrm{d}^{2} \mathrm{~A}_{\mathrm{m}}}{\mathrm{dz}^{2}} \cong \frac{\lambda}{2 \pi \mathrm{n}_{0}} \frac{\mathrm{d}^{2} \mathrm{~A}_{\mathrm{m}}}{\mathrm{dz}},
$$

the second derivatives of $A_{m}$ in Eq. (12) can be neglected. This condition is easily fulfilled in many situations, because it means only that the relative change of $d A_{m} / d z$ over an optical wavelength $\lambda / n_{0}$ in the medium is small. The final form of the $\mathrm{N}$ coupled wave equations becomes then

$$
\frac{d A_{n}}{d z}=\frac{i \mu_{0}}{2 T W_{n} \beta_{n}} e^{i \beta_{n} z} \int_{-\infty}^{+\infty} d x d y \int_{0}^{T} d t e^{-i \omega_{n} t} U_{n}^{*}(x, y) \ddot{p}(x, t)
$$

The physics of the medium is expressed by the relation between the perturbation of the dielectric polarization $\mathbf{p}(\mathbf{x}, \mathrm{t})$ and the total electrical field $\mathbf{E}(\mathbf{x}, \mathbf{t})$, which can formally be written as

$$
\mathbf{p}(\mathbf{x}, \mathrm{t})=\mathrm{F}\{\mathbf{E}(\mathbf{x}, \mathrm{t})\}=\mathrm{F}\left\{\sum_{\mathrm{n}=1}^{\mathrm{N}} \mathrm{A}_{\mathbf{n}}(\mathrm{z}) \mathbf{E}_{\mathrm{n}}(\mathbf{x}, \mathrm{t})\right\}=\mathrm{F}\left\{\sum_{\mathrm{n}=1}^{\mathrm{N}} \mathrm{A}_{\mathrm{n}}(\mathrm{z}) \mathbf{U}_{\mathrm{n}}(\mathrm{x}, \mathrm{y}) \mathrm{e}^{\mathrm{i}\left(\omega_{\mathrm{n}} \mathrm{t}-\beta_{\mathrm{n}} \mathrm{z}\right)}\right\},
$$

where $\mathrm{F}\{\cdot\}$ is an appropriate functional relation to describe the physics. Now it becomes clear, that the $\mathrm{N}$ differential equations for the $A_{n}(z)$ in (14) are coupled through the perturbation $\mathbf{p}(\mathbf{x}, \mathbf{t})$.

In the case that all modes have the same optical frequency $\left(\omega_{n}=\omega\right)$ and $F\{E(x, t)\}$ is linear, $\mathbf{p}(\mathbf{x}, t)$ can be written as $\mathbf{p}(\mathbf{x}, \mathbf{t})=\mathbf{p} \omega(\mathbf{x}) \mathrm{e}^{\mathrm{i} \omega \mathrm{t}}$ and Eq. (14) reduces further to

$$
\frac{d A_{n}}{d z}=\frac{i \omega^{2} \mu_{0}}{2 W_{n} \beta_{n}} e^{i \beta_{n} z} \int_{-\infty}^{+\infty} d x d y U_{n}^{*}(x, y) p_{\omega}(x) .
$$


The integral in Eq. (16) corresponds to a general projection of the perturbation $\mathbf{p}_{\boldsymbol{\omega}}(\mathbf{x})$ onto the $\mathrm{n}$-th mode, which is also known as the scalar product of the two functions. This scalar product involves vectors and complex valued functions of the space coordinates $\mathrm{x}$ and $\mathrm{y}$.

\section{EXAMPLES OF COUPLED WAVE EQUATIONS}

In the following, some typical examples for the application of the coupled wave concept will be presented: volume holograms, acousto-optic modulators, waveguide couplers, polarization effects in optical fibers, and non-linear optics. First, we have to establish the expression for the perturbation $\mathbf{p}(\mathbf{x}, \mathbf{t})$ in each case from the physical properties of the medium. Then, we have to choose a minimum number of appropriate modes $\mathbf{E}_{\mathbf{n}}(\mathbf{x}, \mathbf{t})$ of the wave equation without perturbation. The result is a set of coupled differential equations for the amplitudes $A_{n}(z)$, which can be solved by analytical or numerical integration for specific boundary conditions.

\subsection{Refractive index and absorption (loss or gain)}

The medium without perturbation is transparent, homogenous and isotropic, characterized by $\varepsilon(\mathbf{x})=\mathrm{n}_{0}^{2}$. The perturbation is caused by a change $\Delta \varepsilon$ of the dielectric constant and an electric conductivity $\sigma$, which yields

$$
\mathbf{p}(\mathbf{x}, \mathrm{t})=\varepsilon_{0}\left(\Delta \varepsilon-\mathrm{i} \frac{\sigma}{\omega \varepsilon_{0}}\right) \mathbf{E}(\mathbf{x}, \mathrm{t})
$$

for monochromatic light at frequency $\omega$. As solution without perturbation we choose a single plane wave

$$
\mathbf{E}_{1}(\mathbf{x}, \mathrm{t})=\mathbf{U}_{1} \mathrm{e}^{\mathrm{i}\left(\omega t-\beta_{1} z\right)} \quad \text { with } \quad \beta_{1}=\mathrm{n}_{0} \mathrm{k}=\mathrm{n}_{0} \frac{\omega}{\mathrm{c}} .
$$

Following Eq. (8), the solution with perturbation becomes then

$$
\mathbf{E}(\mathbf{x}, \mathrm{t})=\mathrm{A}_{1}(\mathrm{z}) \mathbf{E}_{1}(\mathbf{x}, \mathrm{t})=\mathrm{A}_{1}(\mathrm{z}) \mathbf{U}_{1} \mathrm{e}^{\mathrm{i}\left(\omega \mathrm{t}-\beta_{1} \mathrm{z}\right)},
$$

from which we get through Eq. (14) or (16) the coupled wave equation

$$
\frac{\mathrm{dA}_{1}}{\mathrm{~d}}=-\frac{\mathrm{i} \mu_{0} \varepsilon_{0} \omega^{2}}{2 \mathrm{Tn}_{0} \mathrm{~kW}}\left(\Delta \varepsilon-\mathrm{i} \frac{\sigma}{\omega \varepsilon_{0}}\right) \mathrm{A}_{1}(\mathrm{z}) \int \mathrm{dx} d \mathrm{y} \mathrm{U}_{1}^{*} \mathrm{U}_{1} \int_{\mathrm{T}} \mathrm{dt}=-\frac{\mathrm{ik}}{2 \mathrm{n}_{0}}\left(\Delta \varepsilon-\mathrm{i} \frac{\sigma}{\omega \varepsilon_{0}}\right) \mathrm{A}_{1}(\mathrm{z}),
$$

because the time integral is equal to $\mathrm{T}$ and the space integral is by definition equal to the normalized energy $\mathrm{W}_{1}$, which happens to be infinity in this case of a plane wave as mode. Integration of Eq. (20) with $A_{1}(z=0)=1$ yields

$$
A_{1}(z)=\exp \left\{-\frac{i k}{2 n_{0}}\left(\Delta \varepsilon-i \frac{\sigma}{\omega \varepsilon_{0}}\right) z\right\}
$$

and finally for the solution of the electric field with perturbation

$$
\mathbf{E}(\mathbf{x}, \mathrm{t})=\mathbf{U}_{1} \exp \left\{-\frac{\mathrm{k} \sigma}{2 \mathrm{n}_{0} \varepsilon_{0} \omega} \mathrm{z}\right\} \exp \left\{\mathrm{i}\left[\omega \mathrm{t}-\mathrm{k}\left(\mathrm{n}_{0}+\frac{\Delta \varepsilon}{2 \mathrm{n}_{0}}\right) \mathrm{z}\right]\right\} \text {. }
$$

Equation (22) represents again a plane wave, but with an energy absorption $\alpha$ and a changed refractive index $n=n_{0}+\Delta n$, given by

$$
\alpha=k \sigma / \mathrm{n}_{0} \varepsilon_{0} \omega \quad \text { and } \quad \Delta \mathrm{n}=\Delta \varepsilon / 2 \mathrm{n}_{0} .
$$

The relations (23) can be used to represent the effect refractive index variations, $\Delta n(x, t)$, and loss or gain, $\alpha(x, t)$, by the corresponding perturbation $\mathbf{p}(\mathbf{x}, \mathbf{t})$ of the dielectric polarization of the medium.

\subsection{Phase gratings (volume holograms) 3,4}

Kogelnik has presented in 1969 a coupled wave theory for all types of thick hologram gratings. Here, we shall limit ourselves for the sake of simplicity to pure phase gratings. The extension to gratings with absorption (or gain) is straight forward. The thick phase grating is given by a spatial modulation of the refractive index 


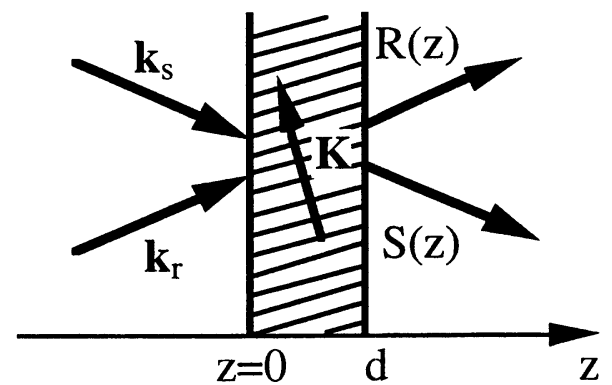

Fig. 2. Geometry for coupled waves through a thick phase grating $\mathbf{K}$.

$$
\mathrm{n}_{1}(\mathbf{x})=\Delta \mathrm{n} \cos (\mathbf{K} \mathbf{x})
$$

The grating vector $\mathbf{K}$ is oriented perpendicular to the fringe planes and is of length $\mathrm{K}=2 \pi / \Lambda$, where $\Lambda$ is the period of the grating (Fig. 2). Following Eqs. (17) and (23), the corresponding perturbation is

$$
\mathbf{p}(\mathbf{x}, \mathrm{t})=2 \varepsilon_{0} \mathrm{n}_{0} \Delta \mathrm{n} \cos (\mathbf{K x}) \mathbf{E}(\mathbf{x}, \mathrm{t}) .
$$

The medium without perturbation is transparent, homogenous and isotropic, characterized by $\varepsilon(\mathbf{x})=\mathrm{n}_{0}^{2}$. As solutions without perturbation we choose two plane waves with wave vectors $\mathbf{k}_{\mathrm{s}}$ and $\mathbf{k}_{\mathrm{r}}$ (Fig. 2) and the same frequency $\omega=\omega_{\mathrm{S}}=\omega_{\mathrm{r}}$, which are represented by

$$
\begin{array}{ll}
\mathbf{E}_{\mathrm{s}}(\mathbf{x}, \mathrm{t})=\mathbf{u}_{\mathrm{s}} \mathrm{e}^{\mathrm{i}\left(\omega \mathrm{t}-\mathbf{k}_{\mathrm{s}} \mathbf{x}\right)}=\mathbf{u}_{\mathrm{S}} \mathrm{e}^{-\mathrm{i}\left(\mathbf{k}_{\mathrm{s}} \mathbf{x}_{\mathrm{H}}\right)} \mathrm{e}^{\mathrm{i}\left(\omega \mathrm{t}-\beta_{\mathrm{s}} \mathrm{z}\right)}, & \beta_{\mathrm{s}}=\left(\mathbf{k}_{\mathrm{s}}\right)_{\mathrm{z}}=\mathrm{n}_{0} \mathrm{ks}_{\mathrm{z}}=\mathrm{n}_{0}(\omega / \mathrm{c}) \mathrm{s}_{\mathrm{Z}}, \\
\mathbf{E}_{\mathrm{r}}(\mathbf{x}, \mathrm{t})=\mathbf{u}_{\mathrm{r}} \mathrm{e}^{\mathrm{i}\left(\omega \mathrm{t}-\mathbf{k}_{\mathrm{r}} \mathbf{x}\right)}=\mathbf{u}_{\mathrm{r}} \mathrm{e}^{-\mathrm{i}\left(\mathbf{k}_{\mathrm{r}} \mathbf{x}_{\mathrm{H}}\right)} \mathrm{e}^{\mathrm{i}\left(\omega \mathrm{t}-\beta_{\mathrm{r}} \mathrm{z}\right)}, & \beta_{\mathrm{r}}=\left(\mathbf{k}_{\mathrm{r}}\right)_{\mathrm{z}}=\mathrm{n}_{0} \mathrm{kr}_{\mathrm{z}}=\mathrm{n}_{0}(\omega / \mathrm{c}) \mathrm{r}_{\mathrm{z}},
\end{array}
$$

where $\mathbf{u}_{\mathbf{S}}$ and $\mathbf{u}_{\mathrm{r}}$ are the unity polarization vectors and $\mathbf{s}$ and $\mathbf{r}$ the unity direction vectors of the two plane waves, respectively. The index $\mathrm{H}$ refers to the hologram plane, which is the plane perpendicular to the $\mathrm{z}$-axes $\left(\mathbf{x}_{\mathrm{H}} \perp \mathrm{z}\right)$, and the index $\mathrm{z}$ indicates the z-component of the corresponding vector. The two selected modes $\mathbf{E}_{\mathbf{S}}(\mathbf{x}, \mathrm{t})$ and $\mathbf{E}_{\mathbf{r}}(\mathbf{x}, \mathrm{t})$ are orthogonal in the sense of Eq. (11), as long as $\mathbf{k}_{\mathrm{S}} \neq \mathbf{k}_{\mathbf{r}}$.

Following Eq. (8), the solution with perturbation can be written as

$$
\mathbf{E}(\mathbf{x}, \mathrm{t})=\mathrm{S}(\mathrm{z}) \mathbf{E}_{\mathrm{S}}(\mathbf{x}, \mathrm{t})+\mathrm{R}(\mathrm{z}) \mathbf{E}_{\mathrm{r}}(\mathbf{x}, \mathrm{t})
$$

from which we get through Eq. (14) or (16) for $R(z)$ the differential equation

$$
\begin{aligned}
\frac{d R}{d z} & =-\frac{i k \Delta n}{W_{r} r_{z}} e^{i\left(\beta_{r} z\right)} \int d x d y u_{r} e^{i\left(k_{r} x_{H}\right)} \cos (K x)\left[u_{r} R(z) e^{-i\left(k_{r} \mathbf{x}\right)}+u_{s} S(z) e^{-i\left(k_{s} \mathbf{x}\right)}\right] \\
& =-\frac{i k \Delta n}{2 W_{r} r_{z}} u_{r} u_{S} S(z) e^{i\left(\beta_{r}-\beta_{s}\right) z}\left[e^{-i K_{z} z} \int d x d y e^{i\left(k_{r}-k_{s}-K\right) x_{H}}+e^{i K_{z} z} \int d x d y e^{i\left(k_{r}-k_{s}+K\right) x_{H}}\right] .
\end{aligned}
$$

Non-vanishing integrals are only obtained if either $\left(\mathbf{k}_{\mathrm{r}}-\mathbf{k}_{\mathrm{s}}-\mathbf{K}\right) \mathbf{x}_{\mathrm{H}}=\mathbf{0}$ or $\left(\mathbf{k}_{\mathrm{r}}-\mathbf{k}_{\mathrm{S}}+\mathbf{K}\right) \mathbf{x}_{\mathrm{H}}=\mathbf{0}$, which means that either the projection $\left(\mathbf{k}_{\mathrm{r}}-\mathbf{k}_{\mathrm{S}}-\mathbf{K}\right)_{\mathrm{H}}$ or $\left(\mathbf{k}_{\Gamma}-\mathbf{k}_{\mathrm{S}}+\mathbf{K}\right)_{\mathrm{H}}$ onto the hologram plane $(\mathbf{x}, \mathrm{y})$ has to be zero. Choosing

$$
\left(\mathbf{k}_{\mathrm{r}}-\mathbf{k}_{\mathrm{S}}-\mathbf{K}\right)_{\mathrm{H}}=\mathbf{0}
$$

corresponds to selecting the +1 st order of diffraction from the thin grating $\cos \left(\mathbf{K x}_{\mathbf{H}}\right)$. We conclude, that the coupled wave concept includes inherently the condition for diffraction. However, there remains in $\mathrm{Eq}$. (29) a phase mismatch $\Delta \mathrm{k}$ in the $\mathrm{z}$-direction, which is

$$
\Delta \mathrm{k}=\left(\beta_{\mathrm{r}}-\beta_{\mathrm{s}}-\mathrm{K}_{\mathrm{Z}}\right)=\left(\mathbf{k}_{\mathrm{r}}-\mathbf{k}_{\mathrm{s}}-\mathbf{K}\right)_{\mathrm{Z}}
$$

Using this phase mismatch $\Delta \mathrm{k}$, we get finally for the two coupled wave equations

$$
\frac{\mathrm{dR}}{\mathrm{d} z}=-\frac{\mathrm{iku_{ \textrm {rs } } \Delta \mathrm { n }}}{2 \mathrm{r}_{\mathrm{z}}} \mathrm{S}(\mathrm{z}) \mathrm{e}^{+\mathrm{i} \Delta \mathrm{kz}}, \quad \frac{\mathrm{dS}}{\mathrm{d}}=-\frac{\mathrm{iku_{ \textrm {rs } } \Delta \mathrm { n }}}{2 \mathrm{~s}_{\mathrm{z}}} \mathrm{R}(\mathrm{z}) \mathrm{e}^{-\mathrm{i} \Delta \mathrm{kz}},
$$

where $u_{r s}=u_{r} \mathbf{u}_{s}$ depends on the polarization of the coupled waves. As we see from Eq. (31), we get maximum transfer of the energy while integrating along the propagation direction $\mathrm{z}$ if $\Delta \mathrm{k}=0$, which is known as Bragg condition. Fulfilling the condition for diffraction and the Bragg condition corresponds to the conservation of the total momentum, $\left(\mathbf{k}_{\mathrm{r}}-\mathbf{k}_{\mathrm{S}}-\mathbf{K}\right)=\mathbf{0}$.

Solving the coupled wave equations (31) with appropriate boundary conditions leads to the well known relations for the diffraction efficiency of phase holograms in transmission or in reflection (Lippman holograms, Bragg mirrors).

\subsection{Acousto-optic modulator 5,6}

An acoustic wave of frequency $\Omega$ and wave vector $\mathbf{K}$ induces a traveling phase grating 


$$
\mathrm{n}_{1}(\mathbf{x})=\Delta \mathrm{n} \cos (\Omega \mathrm{t}-\mathbf{K x})
$$

in the material. Following Eqs. (17) and (23), the corresponding perturbation is

$$
\mathbf{p}(\mathbf{x}, \mathrm{t})=2 \varepsilon_{0} \mathrm{n}_{0} \Delta \mathrm{n} \cos (\Omega \mathrm{t}-\mathbf{K} \mathbf{x}) \mathbf{E}(\mathbf{x}, \mathrm{t}) .
$$

The medium without perturbation is assumed to be transparent, homogenous and isotropic, characterized by $\varepsilon(\mathbf{x})=n_{0}^{2}$.

Compared with the phase grating of the volume hologram in Eq. (24), the acoustic wave brings an additional time variation into the perturbation $\mathbf{p}(\mathbf{x}, \mathbf{t})$. Therefore, we choose as solutions without perturbation two plane waves

$$
\begin{array}{ll}
\mathbf{E}_{\mathrm{S}}(\mathbf{x}, \mathrm{t})=\mathbf{u}_{\mathrm{S}} \mathrm{e}^{\mathrm{i}\left(\omega_{\mathrm{S}} \mathrm{t}-\mathbf{k}_{\mathrm{S}} \mathbf{x}\right)}=\mathbf{u}_{\mathrm{S}} \mathrm{e}^{-\mathrm{i}\left(\mathbf{k}_{\mathrm{S}} \mathbf{x}_{\mathrm{H}}\right)} \mathrm{e}^{\mathrm{i}\left(\omega_{\mathrm{S}} \mathrm{t}-\beta_{\mathrm{s}} \mathrm{z}\right)}, & \beta_{\mathrm{S}}=\left(\mathbf{k}_{\mathrm{S}}\right)_{\mathrm{Z}}=\mathrm{n}_{0} \mathrm{k} \mathrm{s}_{\mathrm{Z}}=\mathrm{n}_{0}\left(\omega_{\mathrm{S}} / \mathrm{c}\right) \mathrm{s}_{\mathrm{Z}}, \\
\mathbf{E}_{\mathrm{r}}(\mathbf{x}, \mathrm{t})=\mathbf{u}_{\mathrm{r}} \mathrm{e}^{\mathrm{i}\left(\omega_{\mathrm{r}} \mathrm{t}-\mathbf{k}_{\mathrm{r}} \mathbf{x}\right)}=\mathbf{u}_{\mathrm{r}} \mathrm{e}^{-\mathrm{i}\left(\mathbf{k}_{\mathrm{r}} \mathbf{x}_{\mathrm{H}}\right)} \mathrm{e}^{\mathrm{i}\left(\omega_{\mathrm{r}} \mathrm{t}-\beta_{\mathrm{r}} \mathrm{z}\right)}, & \beta_{\mathrm{r}}=\left(\mathbf{k}_{\mathrm{r}}\right)_{\mathrm{Z}}=\mathrm{n}_{0} \mathrm{kr}_{\mathrm{Z}}=\mathrm{n}_{0}\left(\omega_{\mathrm{r}} / \mathrm{c}\right) \mathrm{s}_{\mathrm{Z}},
\end{array}
$$

with different frequencies $\omega_{\mathrm{S}}$ and $\omega_{\mathrm{r}}$, respectively. The two selected modes $\mathbf{E}_{\mathbf{S}}(\mathbf{x}, \mathbf{t})$ and $\mathbf{E}_{\mathrm{r}}(\mathbf{x}, \mathbf{t})$ are again orthogonal in the sense of Eq. (11).

Following Eq. (8), the solution with perturbation can be written as

$$
\mathbf{E}(\mathbf{x}, \mathrm{t})=\mathrm{S}(\mathrm{z}) \mathbf{E}_{\mathbf{S}}(\mathbf{x}, \mathrm{t})+\mathrm{R}(\mathrm{z}) \mathbf{E}_{\mathbf{r}}(\mathbf{x}, \mathrm{t}),
$$

from which we get through Eq. (14) for R(z) the differential equation

$$
\begin{aligned}
& \frac{d R}{d}=\frac{i \mu_{0} \varepsilon_{0} n_{0} \Delta n}{T_{W_{r}} \beta_{r}} e^{i\left(\beta_{r} z\right)} \int d x d y \int_{T} d t u_{r} e^{-i\left(\omega_{r} t-k_{r} x_{H}\right)} \frac{\partial^{2}}{\partial t^{2}}\left\{\cos (\Omega t-K x)\left[R(z) E_{r}(x, t)+S(z) E_{S}(x, t)\right]\right\}
\end{aligned}
$$

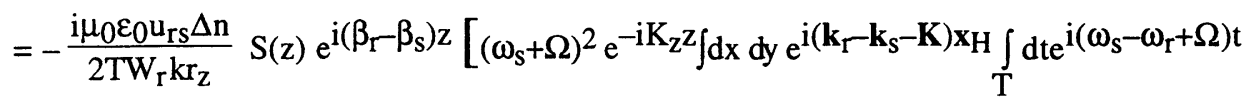

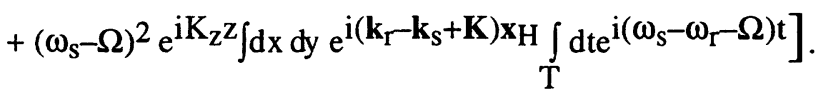

Compared with a stationary phase grating, like in a volume hologram, an additional condition for the frequencies has to be fulfilled to get non-vanishing integrals in Eq. (36). The two possibilities are

$$
\begin{array}{llll}
+1 \text { st order diffraction: } & \left(\mathbf{k}_{\mathrm{r}}-\mathbf{k}_{\mathrm{S}}-\mathbf{K}\right)_{\mathrm{H}}=\mathbf{0} & \text { and } & \left(\omega_{\mathrm{r}}-\omega_{\mathrm{S}}-\Omega\right)=0, \\
\text {-1st order diffraction: } & \left(\mathbf{k}_{\mathrm{r}}-\mathbf{k}_{\mathrm{s}}+\mathbf{K}\right)_{\mathrm{H}}=\mathbf{0} & \text { and } & \left(\omega_{\mathrm{r}}-\omega_{\mathrm{S}}+\Omega\right)=0 .
\end{array}
$$

The condition for the frequencies corresponds to the Doppler shift due to the traveling acoustic wave grating. We conclude, that the coupled wave concept includes also the conservation of the energy in the photon-phonon picture. However, there remains in Eq. (29) a phase mismatch $\Delta \mathrm{k}$ in the z-direction, which is

$$
\Delta \mathrm{k}=\left(\beta_{\mathrm{r}}-\beta_{\mathrm{s}}-\mathrm{K}_{\mathrm{z}}\right)=\left(\mathbf{k}_{\mathrm{r}}-\mathbf{k}_{\mathrm{s}}-\mathbf{K}\right)_{\mathrm{z}}
$$

Using this phase mismatch $\Delta k$, we get finally for the two coupled wave equations

$$
\frac{\mathrm{dR}}{\mathrm{d}}=-\frac{\mathrm{ik} \Delta \mathrm{n}}{2 \mathrm{r}_{\mathrm{z}}} \mathrm{S}(\mathrm{z}) \mathrm{e}^{+\mathrm{i} \Delta \mathrm{kz}}, \quad \frac{\mathrm{d} \mathrm{S}}{\mathrm{d}}=-\frac{\mathrm{ik} \Delta \mathrm{n}}{2 \mathrm{~s}_{\mathrm{z}}} \mathrm{R}(\mathrm{z}) \mathrm{e}^{-\mathrm{i} \Delta \mathrm{kz}},
$$

where we have assumed that $u_{r s}=u_{r} u_{s} \cong 1,|\mathbf{K}| \ll k$, and $\Omega \ll \omega$. Equation (39) is similar to Eq. (31). As we see from Eq. (39), we get maximum transfer of the energy while integrating along the propagation direction $z$ if $\Delta k=0$, which is again the well known Bragg condition. Fulfilling all conditions for diffraction and the Bragg condition corresponds to the conservation of the total momentum, $\left(\mathbf{k}_{\mathrm{r}}-\mathbf{k}_{\mathrm{s}}-\mathbf{K}\right)=\mathbf{0}$ and the energy $\left(\omega_{\mathrm{r}}-\omega_{\mathrm{s}}-\Omega\right)=0$ in he photon-phonon picture.

\subsection{Coupled waves in integrated optics 7}

As an example of coupled waves in integrated optics we take the coupling between two parallel rectangular wave-guides on a planar substrate, as shown in Fig. 3. We assume that the wave-guides are single mode. As the solutions without perturbation we consider the fundamental TE mode $\mathrm{E}_{1}(\mathbf{x}, \mathrm{t})$ in the first wave-guide, $\mathrm{n}_{1}(\mathrm{x})$, and the fundamental $\mathrm{TE}$ mode $\mathrm{E}_{2}(\mathbf{x}, \mathrm{t})$ in the second wave-guide, $\mathrm{n}_{2}(\mathrm{x})$, respectively. The direction of propagation is $\mathrm{z}$ and the confinement in the $\mathrm{y}$-direction is neglected. 

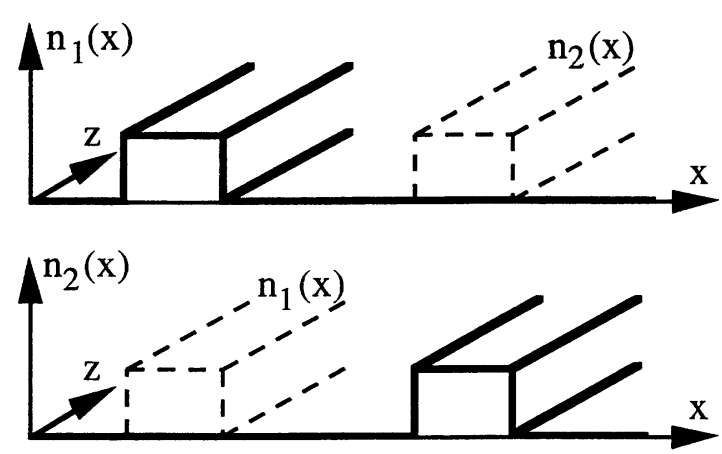

Fig. 3. Geometry for the coupling between to parallel rectangular wave-guides.
Therefore, the two selected modes are represented in the scalar form

$$
\begin{array}{ll}
E_{1}(x, t)=U_{1}(x) e^{i\left(\omega t-\beta_{1} z\right)}, & \left(\beta_{1}=N_{1} k\right), \\
E_{2}(x, t)=U_{2}(x) e^{i\left(\omega t-\beta_{2} z\right)}, & \left(\beta_{2}=N_{2} k\right),
\end{array}
$$

where the mode shapes, $U_{1}(x)$ and $U_{2}(x)$, and the effective refractive indices, $\mathrm{N}_{1}$ and $\mathrm{N}_{2}$ are obtained from wave-guide theory.

The perturbation for the solution $E_{1}$ is the presence of the guide $\mathrm{n}_{2}(\mathrm{x})$ and vice versa, ${ }^{8}$ which leads to

$$
p(x, t)=2 \varepsilon_{0}\left[N_{1} n_{2}(x) E_{1}(x, t)+N_{2} n_{1}(x) E_{2}(x, t)\right],
$$

where the relation $\Delta \varepsilon=2 n_{0} \Delta n$ from Eq. (23) has been used.

Following Eq. (8), the solution with perturbation can be written as

$$
E(x, t)=A_{1}(z) E_{1}(x, t)+A_{2}(z) E_{2}(x, t),
$$

from which we get through Eq. (14) or (16) the coupled wave equations

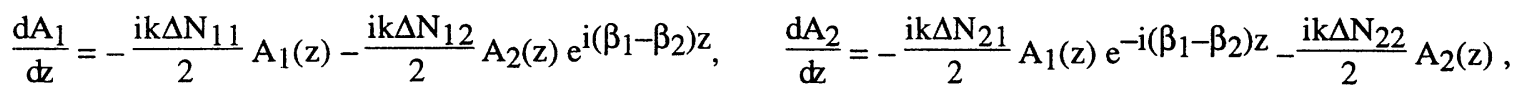

with the coupling integrals

$$
\Delta N_{11}=\frac{2}{W_{1}} \int d x n_{2}(x) U_{1}^{2}(x), \quad \Delta N_{12}=\frac{2 N_{2}}{N_{1} W_{1}} \int d x U_{1}(x) n_{1}(x) U_{2}(x), \quad \text { etc. }
$$

Again the find a phase mismatch term $\Delta \beta=\beta_{1}-\beta_{2}$, which governs the transfer efficiency during propagation (integration along $\mathrm{z}$ ). The coefficients $\Delta \mathrm{N}_{11}$ and $\Delta \mathrm{N}_{22}$ introduce a change of the effective refractive index $\left(\Delta \mathrm{N}_{1}=\Delta \mathrm{N}_{11} / 2\right)$, as discussed in chapter 3.1. The coefficients $\Delta \mathrm{N}_{12}$ and $\Delta \mathrm{N}_{21}$ are responsible for the energy transfer between to waveguides, similar to the coupling by a phase grating discussed in chapter 3.2.

\subsection{Polarization effects in optical fibers 9,10}

An ideal optical fiber has perfect circular symmetry. The polarizations are completely degenerate. Imperfections during the fabrication process may introduce anisotropies, which are mostly of a linear or Cartesian type. Sometimes, large linear anisotropies are introduced on purpose, either by modified core geometry or by mechanical stress, to get linear polarization maintaining fibers, also called high-birefringence or hi-bi fibers. Bending and squeezing optical fibers does also introduce linear birefringence. Rotational effects of polarization occur in twisted fibers (mechanical torsion) and due to the Faraday effect. Coupled wave theory is most adequate to study the changes of polarization of light propagating in single-mode fibers caused by perturbations of geometry or material properties. To describe the superposition of different perturbations it is most suitable to represent each perturbation locally by its respective dielectric tensor. This approach is also well adapted for numerical solutions in the case of arbitrarily distributed perturbations along the fiber.

For a linear birefringence, the perturbation is given by the dielectric tensor

$$
\Delta \varepsilon(\mathrm{z})=\left(\begin{array}{ccc}
\Delta \varepsilon_{1} & \Delta \varepsilon_{6} & 0 \\
\Delta \varepsilon_{6} & \Delta \varepsilon_{2} & 0 \\
0 & 0 & 0
\end{array}\right) \quad \text { with } \quad \begin{aligned}
& \Delta \varepsilon_{1}=\mathrm{n}_{0} \Delta \mathrm{n}(\mathrm{x}) \cos \{2 \phi(\mathrm{z})\} \\
& \Delta \varepsilon_{2}=-\mathrm{n}_{0} \Delta \mathrm{n}(\mathrm{x}) \cos \{2 \phi(\mathrm{z})\} \\
& \Delta \varepsilon_{6}=\mathrm{n}_{0} \Delta \mathrm{n}(\mathrm{x}) \sin \{2 \phi(\mathrm{z})\}
\end{aligned}
$$

where $\Delta \mathrm{n}$ is the difference of the refractive index for the slow and the fast axes of the birefringence and $\phi$ is the orientation of the slow axis with respect to the $\mathrm{x}$-axis. As indicated in Eq. (45) the birefringence $\Delta \mathrm{n}$ and the orientation $\phi$ may change along the fiber axes $z$. In the case of a spun fiber (birefringent fiber spun during the drawing process), $\phi(z)=\alpha z$, where $\alpha$ is the spun rate, and $\Delta \mathrm{n}$ is constant. In the case of linear anisotropy introduced by bending or squeezing an optical fiber, one gets $\Delta \varepsilon$ from evaluation of the photo-elastic or piezo-optic effect.

Optical activity, or circular birefringence, is produced in optical fibers by mechanical torsion as a result of twisting the fiber. The perturbation is then given by the dielectric tensor 


$$
\Delta \varepsilon(\mathrm{x}, \mathrm{y})=\left(\begin{array}{ccc}
0 & 0 & \Delta \varepsilon_{5} \\
0 & 0 & \Delta \varepsilon_{4} \\
\Delta \varepsilon_{5} & \Delta \varepsilon_{4} & 0
\end{array}\right) \quad \text { with } \quad \Delta \varepsilon_{4}=\mathrm{n}_{0}^{2} \mathrm{~g} \tau \mathrm{x} \quad \text { and } \quad \Delta \varepsilon_{5}=\mathrm{n}_{0}^{2} \mathrm{~g} \tau \mathrm{y},
$$

where $\tau=\mathrm{d} \theta / \mathrm{dz}$ is the mechanical torsion and $\mathrm{g}$ is the elasto-optic coupling coefficient ( $\mathrm{g}=0.16$ for silica fibers). Note that the components $\Delta \varepsilon_{4}$ and $\Delta \varepsilon_{5}$ increase with the distance from the center of the fiber.

The Faraday effect is an optical activity induced by the component of a magnetic field in the direction of the light propagation. The corresponding perturbation is given by the dielectric tensor

$$
\Delta \varepsilon=\left(\begin{array}{ccc}
0 & \left(\Delta \varepsilon_{6}\right)^{*} & 0 \\
\Delta \varepsilon_{6} & 0 & 0 \\
0 & 0 & 0
\end{array}\right) \quad \text { with } \quad \Delta \varepsilon_{6}=2 \mathrm{in}_{0} \mathrm{VH}_{\mathrm{z}} / \mathrm{k}
$$

where $\mathrm{V}$ is the Verdet constant and $\mathrm{H}_{\mathrm{z}}$ is the magnetic field component along $\mathrm{z}$.

In weakly guiding single-mode fibers with circular symmetry the electric field distribution can be approximated by two orthogonal linearly polarized modes of the form

$$
\mathbf{U}_{1}(\mathrm{x}, \mathrm{y})=\left(\begin{array}{c}
\mathrm{U}(\mathrm{r}) \\
0 \\
-(\mathrm{i} / \beta) \cos \varphi \mathrm{d} \mathrm{U} / \mathrm{dr}
\end{array}\right), \quad \mathbf{U}_{2}(\mathrm{x}, \mathrm{y})=\left(\begin{array}{c}
0 \\
\mathrm{U}(\mathrm{r}) \\
-(\mathrm{i} / \boldsymbol{\beta}) \sin \varphi \mathrm{dU} / \mathrm{dr} r
\end{array}\right),
$$

with $x=r \cos \varphi$ and $y=r \sin \varphi$. These modes are chosen as the two orthogonal solutions without perturbation. Note that both modes have also a longitudinal component, which turns out to be important for the calculation of the optical activity induced by mechanical torsion. The radial distribution $U(r)$ can be sufficiently well approximated for most fiber profiles by a Gaussian.

Following Eq. (8), the solution with perturbation can now be written as

$$
\mathbf{E}(\mathbf{x}, \mathrm{t})=A_{1}(z) \mathbf{U}_{1}(x, y) e^{i(\omega t-\beta z)}+A_{2}(z) \mathbf{U}_{2}(x, y) e^{i(\omega t-\beta z)},
$$

where $\beta=n_{0} k$ and $n_{0}$ is the effective index of refraction of the fundamental mode in the fiber without perturbation. From Eq. (49) we get through Eq. (14) or (16) for the amplitudes $A_{1}(z)$ and $A_{2}(z)$ the coupled wave equations

$$
\mathrm{dA} / \mathrm{dz}=\sum_{\mathrm{n}=1}^{2} \mathrm{i} \kappa_{\mathrm{nm}}(\mathrm{z}) \mathrm{A}_{\mathrm{m}}(\mathrm{z})
$$

with the coupling coefficients

$$
\kappa_{\mathrm{nm}}(\mathrm{z})=\frac{-\mathrm{k}}{2 \mathrm{n}_{0} \mathrm{~W}_{\mathrm{n}}} \int \mathrm{dx} d \mathrm{y} \mathbf{U}_{\mathrm{n}}^{*}(\mathrm{x}, \mathrm{y}) \Delta \varepsilon(\mathrm{x}, \mathrm{y}, \mathrm{z}) \mathbf{U}_{\mathrm{m}}(\mathrm{x}, \mathrm{y}) .
$$

Integrating Eq. (50) with appropriate initial conditions allows to calculate the development of a particular input polarization all along the fiber length $\mathrm{z}$. The perturbations are given through the dielectric tensor $\Delta \varepsilon$, which may in general depend on $\mathrm{x}, \mathrm{y}$ and $z$. It can be shown, ${ }^{9}$ that the Jones $\mathrm{N}$-matrices ${ }^{11}$ are related to the coupling coefficients of Eq. (51) by $\mathrm{N}_{\mathrm{nm}}=\mathrm{i} \kappa_{\mathrm{nm}}$. If several perturbations are present simultaneously, the corresponding coupling coefficients are obtained by linear superposition.

3.6 Nonlinear optics (parametric coupling) 12,13

The nonlinear dielectric polarization in a birefringent crystal can be described by

$$
p_{i}(x, t)=\varepsilon_{0} \chi_{i k n}^{(2)} E_{k}(x, t) E_{n}(x, t),
$$

where $\chi_{\mathrm{ikn}}^{(2)}$ is the second-order susceptibility tensor. The summations in Eq. (527) run over all three components $(\mathrm{k}, \mathrm{n})$ of the electric field vector $\mathbf{E}(\mathbf{x}, \mathbf{t})$. The medium without perturbation is a birefringent crystal. For a given direction of propagation there are always to orthogonal linear polarizations (eigen-polarizations) which propagate independently and are characterized by two different indices of refraction. We shall therefore choose as solutions without perturbation three collinear plane waves 


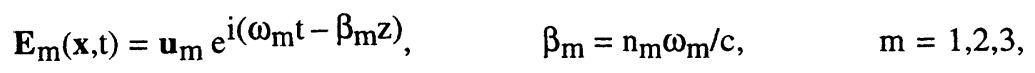

corresponding to these eigen-polarizations $\mathbf{u}_{\mathrm{m}}$, but at different optical frequencies $\omega_{\mathrm{m}}$. Note that whenever nonlinear effects are involved, we have to consider the electric field $\mathbf{E}(\mathbf{x}, \mathbf{t})$ as a real valued (physical) quantity. Therefore, the solution with perturbation takes the form

$$
\mathbf{E}(\mathbf{x}, \mathrm{t})=\frac{1}{2} \sum_{\mathrm{m}=1}^{3}\left[\mathrm{~A}_{\mathrm{m}}(\mathrm{z}) \mathbf{E}_{\mathrm{m}}(\mathrm{z}, \mathrm{t})+\mathrm{A}_{\mathrm{m}}^{*} \mathbf{E}_{\mathrm{m}}^{*}(\mathrm{z}, \mathrm{t})\right] .
$$

from which we get through Eq. (14) and after spatial integration for the amplitude $\mathrm{A}_{3}(\mathrm{z})$ the differential equation

$$
\frac{\mathrm{dA}_{3}}{\mathrm{~d} z}=\frac{\mathrm{i} \mu_{0} \varepsilon_{0}}{2 \mathrm{~T} \beta_{3}} \mathrm{e}^{\mathrm{i} \beta_{3} \mathrm{z}} \int_{\mathrm{T}} \mathrm{dt} \mathrm{u}_{3 \mathrm{i}} \chi_{\mathrm{ikn}}^{(2)} \mathrm{e}^{-\mathrm{i} \omega_{3} \mathrm{t}} \frac{\partial^{2}}{\partial \mathrm{t}^{2}}\left[\mathrm{E}_{\mathrm{k}}(\mathrm{z}, \mathrm{t}) \mathrm{E}_{\mathrm{n}}(\mathrm{z}, \mathrm{t})\right], \quad \mathrm{i}, \mathrm{k}, \mathrm{n}=\mathrm{x}, \mathrm{y}, \mathrm{z},
$$

where $E_{k}(z, t)$ and $E_{n}(z, t)$ are the components of the electric field vector $E(x, t)$ in Eq. (54) and $u_{3 i}$ are the components of the polarization vector $\mathbf{u}_{3}$. Similar equations are also obtained for $\mathrm{A}_{1}(\mathrm{z})$ and $\mathrm{A}_{2}(\mathrm{z})$.

From the products $E_{k}(z, t) E_{n}(z, t)$ we get all possible combinations of the frequencies $\omega_{m}: \omega_{1}+\omega_{2}, \omega_{2}+\omega_{3}, \omega_{3}+\omega_{1}$; $\omega_{1}-\omega_{2}, \omega_{2}-\omega_{3}, \omega_{3}-\omega_{1}$; etc. All these frequencies are present in the non-linear dielectric polarization $\mathbf{p}(\mathbf{x}, \mathbf{t})$. Non-vanishing integrals over the time in Eq. (55) are only obtained when $\omega_{3}$ is equal to one of these sum- or difference-frequencies. We choose $\omega_{3}=\omega_{1}+\omega_{2}$, which leads to the coupled wave equations

$$
\begin{aligned}
& \mathrm{dA}_{1} / \mathrm{dz}=-\left(i \omega_{1} / 2 \mathrm{n}_{1} \mathrm{c}\right) \kappa_{123} \mathrm{~A}_{2}^{*} \mathrm{~A}_{3} \mathrm{e}^{\mathrm{i} \Delta \mathrm{kz}}, \\
& \mathrm{dA}_{2} / \mathrm{dz}=-\left(\mathrm{i} \omega_{2} / 2 \mathrm{n}_{2} \mathrm{c}\right) \kappa_{213} \mathrm{~A}_{1}^{*} \mathrm{~A}_{3} \mathrm{e}^{\mathrm{i} \Delta \mathrm{kz}}, \\
& \mathrm{dA}_{3} / \mathrm{dz}=-\left(\mathrm{i} \omega_{3} / 2 \mathrm{n}_{3} \mathrm{c}\right) \kappa_{312} A_{1} A_{2} \mathrm{e}^{-\mathrm{i} \Delta \mathrm{kz},}
\end{aligned}
$$

with the phase mismatch term

$$
\Delta \mathrm{k}=\beta_{1}+\beta_{2}-\beta_{3}=\left(\mathrm{n}_{1} \omega_{1}+\mathrm{n}_{2} \omega_{2}-\mathrm{n}_{3} \omega_{3}\right) / \mathrm{c} \quad \text { and } \quad \omega_{1}+\omega_{2}-\omega_{3}=0 .
$$

The nonlinear coupling coefficients $\kappa_{\mathrm{abc}}$ are given by

$$
\kappa_{\mathrm{abc}}=\mathrm{u}_{\mathrm{ai}} \chi_{\mathrm{ikn}}^{(2)} \mathrm{u}_{\mathrm{bk}} \mathrm{u}_{\mathrm{cn}}, \quad \mathrm{a}, \mathrm{b}, \mathrm{c}=1,2,3, \quad \mathrm{i}, \mathrm{k}, \mathrm{n}=\mathrm{x}, \mathrm{y}, \mathrm{z},
$$

where $u_{a i}$, $u_{b k}$, and $u_{c n}$ are the Cartesian components of the eigen-polarization vectors $\mathbf{u}_{\mathrm{a}}$, $\mathbf{u}_{\mathrm{b}}$, and $\mathbf{u}_{\mathrm{c}}$ of the three coupled waves, respectively. A triple sum is taken over $i, k$, and $n$. The equations (56) are the basic relations describing nonlinear parametric interactions.

\section{CONCLUSIONS}

We have developed the general concept of coupled waves to describe the propagation of optical waves in a dielectric medium which can be characterized by any kind of perturbation (spatial and temporal) of the dielectric polarization with respect to a basic situation with known solutions. The concept of coupled waves is a powerful method to find (approximate) solutions of the wave equation (for the electric field vector) assuming a perturbation induced by the dielectric polarization of the medium.

Then, we have presented some typical examples for the application of the coupled wave concept: volume holograms, acousto-optic modulators, waveguide couplers, polarization effects in optical fibers, and non-linear optics. First, we had to establish the expressions for the perturbation in each case from the physical properties of the medium. Then, we had to choose a minimum number of appropriate modes of the wave equation without perturbation. The result is a set of coupled differential equations for the amplitudes of these modes, which can be solved by analytical or numerical integration for specific boundary conditions.

This approach puts the emphasis on teaching concepts rather than presenting particular effects. Conservation of momentum ( $k$, diffraction) and energy ( $\omega$, photons) is intrinsic. The fundamental role of phase-matching (Bragg) and the similarities of the solutions for different physical effects emerge clearly. Coupled waves are based on the concept of modes, which allows to find eigen-functions (supermodes) for the wave propagation in the medium with perturbation. Coupled mode 
equations are also well suited for numerical evaluation. The presented concepts of coupled modes, with a minimum number of basic modes and in the approximation of weak coupling, can be extended to more accurate solutions (e.g. rigorous diffraction theory). These are the reasons why I believe that introducing coupled waves as a concept in modern optics is most adequate for education in optics at a university level.

\section{REFERENCES}

1. A. Yariv, Optical Electronics in Modern Communications, Oxford University Press, New York, 1997.

2. B.E.A. Salech and M.C. Teich, Fundamentals of Photonics, John Wiley \& Sons, New York, 1991.

3. H. Kogelnik, "Coupled wave theory for Thick Hologram Gratings", Bell System Tech. J., 48, 2909-2947 (1969).

4. Ref. 1, chap. 14 , pp. 541-549.

5. Ref. 2, chap. 20 , pp. $799-812$.

6. Ref. 1 , chap. 12 , pp. $474-486$.

7. Ref. 1, chap. 13 , pp. 521-531.

8. W. Streifer, "Coupled mode theory", Electron. Lett., 23, 315-316 (1987).

9. R. Dändliker, "Rotational effect of polarization in optical fibers", Anisotropic and Nonlinear Optical Waveguides, C.G. Someda and G. Stegemann, pp. 39-76, Elsevier Science Publishers, Amsterdam, 1992.

10. R. Ulrich and A. Simon, "Polarization optics of twisted single mode fibers", Appl. Opt., 18, 2241-2251 (1981).

11. R.C. Jones, "A new calculus for the treatment of optical systems. VII. Properties of the N-matrices", J. Opt. Soc. Am., 38, 671-685 (1948).

12. Ref. 1 , chap. 8 , pp. $273-285$.

13. Ref. 2, chap. 19 , pp. $762-774$. 